\title{
Der Monddoktor im Alten Berlin
}

Das „Alte Berlin“ und seine „Berliner“ kann man sich heute kaum noch vorstellen. In ihren Erinnerungen an „Mein liebes altes Berlin“ anno 1912 berichtete die Schriftstellerin Agathe Nalli-Rutenberg von ihrer Berliner Jugendzeit in der ersten Hälfte des 19. Jahrhunderts. Vor dem Anhaltstore (Anhalter Tor) zogen sich weite Wiesenflächen und Felder hin. Es standen nur wenige Gebäude in der Schöneberger Straße; man lebte dort wie aufdem Lande. Die Leute kannten sich alle untereinander. Auf den Wiesen wuchsen im Sommer Blumen die Hülle und Fülle, Butterblumen, weiße Gänseblümchen und die zarten Wiesennelken. Überhaupt war Berlin damals eigentlich eine Gartenstadt. Fast hinter jedem Hause befand sich ein hübscher Garten. Besonders weit ausgedehnt waren diese Gärten in der Friedrichstraße [10]. Gemüsegärten zu beiden Seiten, dazwischen eine Koppel mit grasenden Pferden und spielenden Fohlen. [9].

Dieses idyllische Berlin des frühen 19. Jahrhunderts geriet durch die schnelle Industrialisierung und den Ausbau der Anhalter und Potsdamer Eisenbahnen ab 1838 in den Sog der allzu schnell aufstrebenden Großstadt, was ich selbst aus den Erzählungen der Großeltern noch nacherleben durfte. Es war einerseits das Berlin der prunkvollen Kaiserzeiten von Friedrich III (18311888) und Wilhelm II (1859-1941), aber andererseits auch des Miljöh von Heinrich Zille (1858-1929) mit allergrößten sozialen Problemen der Bevölkerung.

Die industrielle Reform Berlins mit der Verelendung ihrer Einwohner ist als Voraussetzung für die nachfolgenden Ereignisse zu sehen. Ganz am Anfang, so um 1800 herum, lag diese Entwicklung noch im Rahmen eines kulturellen, in der Aufklärung verankerten Rahmens. Dann traten aber die Interessen des Kapitals mehr und mehr in den Vordergrund [5]. Für die immer größer werdenden technischen Projekte wurden unzählige Arbeitskräfte aus dem ganzen Land gesucht. Mein Großvater mütterlicherseits kam als Zimmermann aus Sachsen zu Siemens nach Berlin, der andere Großvater als Schlosser für den Bau der großen Swinemünder Eisenbahnbrücke am Gesundbrunnen aus der Pfalz.
Um 1880 feierte die wissenschaftliche Medizin in der Berliner Charité zwar Welterfolge, die ärztliche Versorgung der einfachen Menschen aber ist auf niedrigsten Niveau stecken geblieben. Mystische und magische Behandlungsmethoden waren gang und gäbe, und Scharlatane konnten leicht ihre Anhänger finden. Zu ihnen gehörten der Ziegenprophet im siebenjährigen Schlesischen Krieg, der barfuß und nur mit einem Ziegenfell bekleidet die Kranken mit dem Straßenkothe behandelte, oder der prophezeihende Weber Pfannenstiel oder der trunksüchtige Berlinische Kuhdoktor Kunath mit seiner mystischen Seife gegen alle Krankheiten der Haut [8]. Ein anderer Scharlatan, der Monddoktor, löste geradezu eine revolutionäre Welle in der Bevölkerung aus, die schließlich ein jähes Ende fand.

\section{Der Monddoktor}

Wer in den Jahren 1780 und 1781 in Berlin war, hörte gewiß auch von den Wunderkuren eines Mannes reden, der anfangs einzig Brüche, hernach aber auch andre Krankheiten durch Mondschein und Gebet unfehlbar heilte, den Personen jedes Geschlechts und jedes Standes haufenweise besuchten, und der viel Glauben fand, zuletzt auch bei Leuten, die ihn nie gesehen, noch weniger hätten prüfen können [1]. So beschrieb der Herausgeber der Berlinischen Monatsschrift Biester die Situation anno 1781.

Die Machenschaften des sogenannten Monddoktors riefen bald die Preußischen Gesundheitsbehörden auf den Plan. Das Oberkollegium medicum bestellte deshalb am 5. April 1781 Dr. Johann Theodor Pyl, Stadtphysikus und Magister für Gerichtliche Arzneywissenschaft, sowie den Hebammenlehrer und Assessor der Chirurgie Herrn Hagen dazu, nähere Erkundigungen einzuziehen. Das Ergebnis wurde dann in der Berlinischen Monatsschrift veröffentlicht [14]. Ein zweiter Bericht mit nahezu demselben Wortlaut stammt von dem seinerzeit bekannten jüdischen Arzt Dr. Marcus Herz vom 9. Juni 1781 [4, 8]. Offensichtlich haben Dr. Pyl und Dr. Herz den Besuch des Mondarztes gemeinsam durchgeführt.
In dem Artikel heißt es weiter [14]: Der Mann, der diese Kuren unternimmt, heißt Weisleder, soll ein Strumpfwirker sein. Anfangs gab er sich nur allein damit ab, itzt aber auch seine Frau, welche die weiblichen Kranken besorgt. Seine ganze Methode besteht darin: Er läßt den Schaden entblößen, und gegen den Mond, der im ersten zunehmenden Viertel sein muß, halten; dann berührt er den Schaden mit der einen Hand, indeß er die andre, nebst dem Gesicht, zum Monde wendet, einige unverständliche Worte murmelt, und dann betet; woraufer die Leute, nachdem er ihnen Glauben zu Gott, der nur allen helfen könne, einzusprechen versucht, fortschickt. Er fordert nie Geld; wenn aber hernach etwas gegeben wird, schlägt er es nicht aus.

Der Pöbel, der alles glaubt, wenn es nur fein wunderbar ist, thut auch hier durch seine Zusätze das Meiste. Selbst die Kranken sind so von Vorurtheilen für diesen Menschen eingenommen, daß sie Besserung glauben, wo in der That keine ist.

\section{Die Leinemacher}

Dr. Pyl vertrat die Ansicht, dass insbesondere die Leineweber, Strumpfwirker, Tuch- und Zeugmacher unter den Wunderheiligen anzutreffen sind. Sie zählten seinerzeit zu den „unehrenhaften Berufen“. Ihr müßiger Geist ist zu nichts Erhablichem fähig, er schweift in Einbildungen und Visionen aus, worin er noch durch das beständige Singen geistlicher Lieder während der Arbeit unterhalten wird. Durch das tagelange Sitzen mit vorgebeugtem Unterleibe werden Eingeweide und Gekröse gedrückt, der Umlauf des Blutes gestört, das Verdauungsgeschäft geschwächt und vorzüglich Blähungen erzeugt, die, wenn sie anstatt den natürlichen Weg zu nehmen, nach oben steigen, den Menschen bald zum Geisterseher, bald zum Wahrsager, und bald zum Doktor machen [14].

\section{Die Wallfahrt zum Mond- doktor}

Am 9. Juni 1781 nachmittags um 5 Uhr hat Dr. Pyl, der Berliner Stadtphysikus, eine Sitzung des Monddoktors besucht [14]. Sie fand an der Kommandantenstraße/ 
Ecke Jakobstraße statt (heute Kreuzberg). Die ganze Gegend wimmelte von Blinden und Hinkenden, die mit einem Eifer und einer Zuversicht in die Jakobstraße sich hineindrängten und hineingeleitet wurden. Überall war man von Krüppeln umgeben.

Von da aus begaben wir uns nach dem Tempel selbst, einem elenden Bierhause von der niedrigsten Klasse. Alles war voll gepfropft von Leuten mit offenbaren und heimlichen Verstümmelungen, von Leuten aller Alter und aller Geschlechter, aller Religionen und aller Stände. Alles unterhielt sich von den stadtkundigen Kuren des Mondpriesters und von der eigenen Erleichterung, die jedes von ihnen an seinem eigenen Uebel seit einigen Tagen spürte. Alles harrte mit einer peinlichen Ungeduld auf die Ankunft des Priesters.

Aber der Doctor verspätete sich, weil er dringend - in einem sechsspännigen Wagen - zur Prinzessin und zum Prinzen gerufen wurde. Am nächsten Tage sah es vor dem Bierhause nicht anders aus. Man musste sich zwischen den prächtigen Equipagen hindurchdrängen. Im Schankraum der Bierkneipe befanden sich wieder einige hundert Menschen.

Die Zu- und Abgänge der Heilsuchenden dauerten von $16^{\circ}$ bis nach Mitternacht, und dabei dauerte jede einzelne Kur nur eine Minute. Es müssen sich tausend täglich hier eingefunden haben: eine ungeheure Anzahl Gebrechliche, aber wahrlich eine weit ungeheurere Zahl Narren im Verhältniß mit der Volksmenge in unserer Stadt! Jedoch handelte es sich nicht um den niedrigsten Pöbel, sondern fast nichts weiter als reich und wohlgekleidete Herren und prächtig aufgesetzte Damen, deren Anzug und Miene Rang und Stand verriethen. Wir sahen ganze Haufen abziehen, die theils die Kur schon genossen, theils unverrichteter Sache zurückgingen, weil sie nicht durchkonnten; aber eben so viele Haufen kamen immer von neuem wieder an.

\section{Der Ablauf der Kur}

Dr. Pyl reihte sich in die Schlange der Teilnehmer an einer Kur ein [14]. An dem einen Ende der (Bier-)Stube war eine Treppe, die nach dem zweiten Stockwerke des Hauses, dem eigentlichen Sitze der Gottheit führte. Ein wachhabender Soldat ließ immer nur zu zwölfen auf einmal ein und aus. Auch war eine

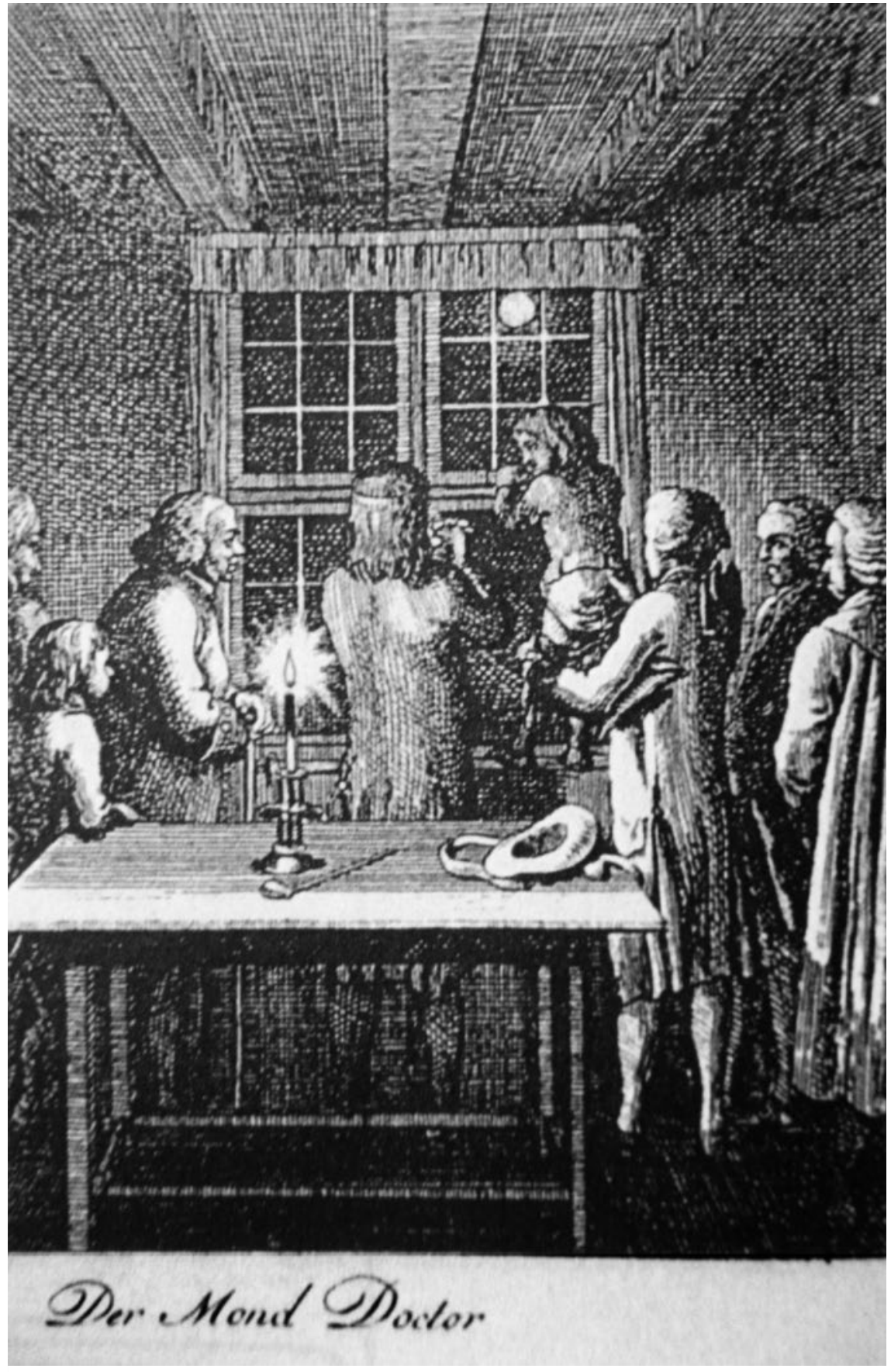

Abb. 1 Der Monddoktor. Zeichnung von Daniel Chodowiecki (1721-1801) in Serie Modetorheiten. Der Leistenbruch des 4-jährigen Jungen wird mit geöffneter Hose vom Vater dem Mond entgegengehalten. Links daneben murmelt der Monddoktor ein Gebet. (Quelle: Daniel Chodowieki [1726-1801] - Serie Modetorheiten: Der Mond Doctor; Berliner Monatsschrift, April 1783, S. 382)

Art systematischer Ordnung in der Kur, indem nicht alle Krankheiten untereinander, sondern klassenweise zur Behandlung zugelassen wurden, erst die Brüche, dann die Blindheiten, die Taubheiten u.s.w. Die Wache rief nun gerade: „Sind die Brüche nun alle? Die Tauben herauf!“
Auf dem oberen Flur befanden sich zwei Stuben. In der einen befanden sich die Damen. Davor stand ein Mädchen mit Billets: „Sie können dafür bezahlen, was beliebt, aber unter zwei Groschen wird nicht angenommen." 
Die Männer gingen in den anderen Raum. Als wir hereinkamen, fand ich eine leere, schmutzige, niedrige Stube eines gemeinen Handwerkers. Ein großer Tisch in der Mitte, ein paar Schemmel an der Wand machte alles Meuble aus. Wir standen da, unserer zwölf, voller Erwartung und Ehrfurcht, bis auf einen einzigen bejahrten, langen, hagern Mann mit ausgekämmten Haaren, einem blauen groben Kleide, der ganz ungenirt mit seiner Tabakspfeife in der Stube herumging. Und dies war der Doktor selbst. Seine Physiognomie war eine von der gemeinsten (unauffälligen) Art, weder die eines Betrügers noch eines Betrogenen. Der Mann schien so wenig auf sein Werk einen Werth zu legen, als sich dessen zu schämen; sondern er that so gleichgültig in allen seinen Gebehrden, als wenn er etwas ganz Alltägliches verrichtete.

Nun legte er die Pfeife nieder. „Wir wollen nun dabei gehen, es wird spät. "Darauf öffnete er das Fenster, und eine nach unten fallende Markise verhinderte, daß vom Garten herauf die Operationen nicht mit angesehen werden konnten. Darauf rief er einen sehr hübschen Knaben von ungefähr vier Jahren zu sich, der von seinem Vater, einem jungen dem Scheine nach wohlhabenden und vernünftigen Manne geführt ward, stellte ihn zur linken Seite aufs Fenster, öffnete ihm die Beinkleider, nahm das (Bruch-) Band herunter, legte die linke Hand auf die verletzte Stelle, während er den Mond, der in der That gar nicht zu sehen war, anzugaffen schien, und einige Worte murmelte ( $\triangleright \boldsymbol{A} \boldsymbol{b} \boldsymbol{b} . \mathbf{1})$. Darauf faltete er beide Hände gegen den Mond, sprach wiederum einige leise Worte, und damit war es aus. Er setzte den Knaben herunter, der Vater nahm ihn auf die Seite und verband ihn wiederum; und dieser gesetzte Mann fragte das Kind: "Wie ist es, mein Sohn, thut es noch so wehe wie vorher?" „O ja, Papa, “ erwiderte dieser.

Mit den übrigen sechsen, die alle Brüchige waren, verfuhr er auf die erwähnte Weise. Er nahm dann den tauben Burschen aus meiner Gesellschaft vor, dem er erst die Hand aufs Ohr legte, hernach in dasselbe etwas hineinflüstert, und dann sein Gebet verrichtete. Endlich kam die Reihe an mich. Da ich keinen offenbaren Schaden aufzuweisen hatte, so gab ich ein gichtiges Reißen in meinem rechten Schenkel vor. „Das kann ich nicht kurieren“, sagte er, „die Leute denken auch, ich kann alles“.

-Tab. 1 Magia naturalis

Teilgebiete der Magia naturalis [11]

Astronomia
Astrologia
Pyromantia
Chaomantia
Hydromantia
Geomatia
Alchimia

Spagyrica
Sternenkunde

Sternendeutung

Wahrsagung aus Feuer

Wetter/Atmosphäre

Wahrsagung aus Wasser

Voraussagung von Sandzeichnungen

Verwandlung von Metallen in Gold und Silber

Abtrennung des geistigen "Arkada" aus Stein
So ging es einer nach dem anderen weiter. Auf einmal sah er bedächtig den Mond an. „Nun müssen wir einhalten, es ist wirklich eine Streife über dem Mond. “ Aber es waren weder Mond noch Streifen zu sehen. Die Frau Doktorin behandelte nach der anderen Seite des Hauses zum Mond hin. Die Kuren mußten drei Tage hintereinander im ersten Mondvirtel wiederholt werden; ja einige Kranke mußten sich denselben wohl drei bis vier Monate hintereinander unterwerfen [14].

\section{Exitus letalis}

Unter dem Titel Casus Nr, 10 wurde von Dr. Pyl die nachfolgende Krankheitsgeschichte mitgeteilt, aufgrund derer die Aktivitäten des Monddoktors ein jähes Ende fanden [14]. Die Gastwirthin Diederich unter den Linden ward vom Professor Voitus („einem vortrefflichen, practischen Chirurgen und Geburtshelfer" [6]) an einem sehr grossen und gefährlichen Nabelbruch mit vieler Mühe kurirt. Vor einigen Monaten trat er so sehr aus, daß er wie ein französisches Zweigroschenbrot groß und sehr inkarcerirt war. Nach zweimal 24 Stunden konnte er zurückgebracht (reponiert) werden; die Oefnung war wie ein Thalerstük groß. Es wurden viele Klystire, erweichende, auflösende, abführende, und andre dienliche Mittel gebraucht; das aufgelegte Eis that noch das meiste. Sie hat seitdem zwar Beschwerden gehabt, aber nur, wenn sie sich zu stark bewegte, blähende Speisen aß, u.s.w. Sie trug beständig das Bruchband mit der gehörigen Pelotte und befand sich wohl dabei. Endlich fiel ihr ein, zum Monddoktor zu gehen, er versprach ihr völlige Hülfe und legte seine Hand auf ihr Bruchband. Nun glaubte sie weder ihrem vorigen Arzte noch dem Bruchbande für ihr itziges Wohlbefinden verbunden zu sein, sondern schrieb alles dem Monde und
Weisleders Wunderhand zu, die sie auch nach Gebührde herausstrich und allen empfahl. Zuletzt war sie so davon überzeugt, daß sie ihr Bruchband wegwarf; aber der Bruch trat sogleich von neuem aus, inkarcerirte, und sie starb elendiglich daran.

\section{Die Magia naturalis}

Seit der Antike versuchen die Menschen unerreichbare Wünsche oder unerklärliche Phänomene auf übernatürliche Erscheinungen zurückzuführen. Zu Beginn der Neuzeit hat sich Paracelsus (1493-1541) in besonderer Weise damit befasst. Er unterschied zwischen der Magie, die auf den Einflüssen des Glaubens beruhte, und der Zauberei, dem Werk des Teufels und der Hexen [11].

Die Magie wurde von verschiedenen Religionen, auch der christlichen Religion, angewendet, um dämonische Kräfte abzuwehren. Magie sollte dem Anwender zum Nutzen gereichen. Im Gegensatz dazu verstößt die Schwarze Magie gegen den Glauben und die aktuellen Sitten, um ein bestimmtes Ziel zu erreichen.

Die Magia naturalis bediente sich verschiedener Erscheinungen in der Natur. Paracelsus differenzierte mehrere Formen ( $\triangleright$ Tab. 1) und setzte sie zur Heilung von Krankheiten ein. Geister und Dämonen kamen darin nicht vor $[3,11]$. Der neapolitanischen Arzt Giambattista della Porta (1535-1615) verfasste anno 1558, also nach Paracelsus, ein 20-bändiges Werk unter dem Titel Magia naturalis [13]. 


\section{Paracelsus und der Mond}

In ausführlicher Weise hat sich Paracelsus mit der Rolle des Mondes in der Medizin befasst [12]. Er schreibt, der kranckheit unnd gesundtheit Samen sind im Leib und müssen wachsen. Darauf hat der Mond seinen Einfluss, so er der Erden zufügt. In diser Geburt ist er windig (schädlich), in der andern naß / jetzt kalt / jetzt warm / und alles der Ein Mond: Aber durch sein newe geburt (Mondaufgang) / wird ihm sein Regiment (Wirksamkeit) durch die imprimierten Ascendenten (Stellung der Sterne) in ein ander Art und Weiß gezogen und gelegt. Den Mond selbst trifft keine Schuld an Krankheit und Gesundheit, sondern nur den Aszendenten. Sowohl beim Sammeln der Arzneipflanzen und bei der Zubereitung von Rezepten als auch bei der ärztlichen Prognostik der Krankheit muss der Arzt diese Faktoren berücksichtigen. Darumb sollend jhr wissen, daß die eussern Sphaera (Gestirne) ist die Schule der gantzen Artzney / nicht Corpora , sonder spiritus (Nicht der Sternenkörper, sondern sein Geist) [12].

\section{Zusammenfassung}

Der Einfluss des Mondes auf Gesundheit und Krankheit wurde seit Menschengedenken hoch angesehen [7]. Früher spielte dabei die Magie eine wichtige Rolle. Anno 1936 hatte ich als Kind von 6 Jahren an den Händen mehrere dieser kleinen juvenilen Wärzchen, die sich manchmal entzündeten und dann schmerzten. Der Großvater wusste Rat. In einer sommerlichen Vollmondnacht gingen wir beide um Mitternacht an die Mistkute hinten im Garten. Ich musste die Hände mit einem feuchten Ledertuch in Richtung zum Vollmond hin gründlich abwischen und den Lappen anschließend über die Schulter wegwerfen. Wir durften uns auf keinen Fall mehr umdrehen und gingen wieder schlafen. Für mich erschien die Situation überaus gruselig. Nach ein paar Tagen waren alle Warzen verschwunden und sind nie wieder aufgetreten.
Zwischen Vollmond und Krankheit gibt es keine wissenschaftlich nachweisbaren Beziehungen. Aber Schlafstörungen in einer Vollmondnacht können wir uns z. B. immer noch nicht ganz erklären. Jedenfalls hat die Studie von Christoph Cajochen anno 2013 entsprechende Daten gebracht und viele Diskussionen ausgelöst [2]. Die Welt um Paracelsus stand überirdischen Einwirkungen sehr aufgeschlossen gegenüber. Paracelsus lebte in der Philosophie der Antike und Renaissance. Die Scharlatanerie des Berliner Monddoktors 300 Jahre nach Paracelsus fällt sofort ins Auge. Den Menschen im 19. Jahrhundert ging es in ärztlicher Hinsicht erbärmlich schlecht. Sie wollten aktiv etwas gegen ihre Krankheiten unternehmen, allerdings ohne jede Kenntnis der Naturwissenschaften. Es blieb ihnen nichts anderes übrig, als sich an den berühmten Strohhalm zu klammern. Schweres Leid führt auch heute den Patienten in verzweifelter Situation zur Magia naturalis, wenn die Schulmedizin am Ende ihrer Weisheit angelangt ist.

\section{Quod nimis miseri volunt, hoc facile cre- dunt \\ Was die sehr Unglücklichen wollen, das glauben sie leicht}

Interessenkonflikt

Die Autoren geben an, dass kein Interessenkonflikt besteht.

\section{Autorinnen/Autoren}

Prof. Dr. Wolfgang Hach

Venenzentrum Frankfurt am Main

\section{Korrespondenzadresse}

Prof. Dr. med. Wolfgang Hach

Tacitusstrasse 66

60439 Frankfurt am Main

E-Mail: hach.frankfurt@gmx.de
Literatur

[1] Biester JE. Der Monddoktor in Berlin. Berlinische Monatsschrift 1 (1783); 353-367

[2] Cajochen C. Evidence that the lunar cycle influendes human sleep. Current Biology 2013; 23:1485-8. https://doi/10.1016/j. cub.2013.06.029 am 13.11.2018

[3] Hach W. Erste Beschreibung der Sympathetischen Salbe durch Paracelsus anno 1622. Phlebologie 2015; 44:37-42

[4] Herz M. Die Wallfahrt zum Monddoktor in Berlin. S. Pyl JT. Berlinische Monatsschrift 1 (1783); 368-384

[5] Klein, U. Humboldts Preußen. Wissenschaft und Technik im Aufbruch. S. 294; 300. Wissenschaftliche Buchgesellschaft. Darmstadt 2015

[6] Knebel IG. Versuch einer chronologischen Uebersicht der Literaturgeschichte der Arzneiwissenschaft zur Beförderung und Erleichterung derselben. S 303. Kern. Breslau 1799

[7] Lebenwaldt A. Land-Stadt-Hauß-ArtzneyBuch. S. 15, 398. Lochner. Nürnberg 1705

[8] Leder C M. Die Grenzgänge des Marcus Herz. Beruf, Haltung und Identität eines jüdischen Arztes gegen Ende des 18. Jahrhunderts. S. 188-196. Waxmann. Münster u. a. 2007

[9] Ludwig H. Altberliner Bilderbogen. S. 29. Groszer. Berlin 1965

[10] Nalli-Rutenberg A. Mein liebes, altes Berlin. S. 19. Continent. Berlin 1912

[11] Paracelsus. Opera, Bücher und Schrifften / so viel deren zur Hand gebracht. S. 298, 311, 549. Zetzner. Straßburg 1603

[12] Paracelsus. Chirurgische Bücher und Schrifften. S. 222 ff. Zetzner. Straßburg 1618

[13] Porta della G. Magia naturalis. Deutsche Übersetzung. Erstes Buch. Joanne Baptista Porta. Natürliche Magia. Rauschern. Magdeburg 1512. Permalink: http://diglib.hab. de/drucke/115-2-phys/start.htm am 31.10.2018

[14] Pyl JT. Die Wallfahrt zum Monddoktor in Berlin. Berlinische Monatsschrift 1 (1783); 368-384

Bibliografie

DOI https://doi.org/10.1055/a-0833-1199 Phlebologie 2019; 48: 11-14 (c) Georg Thieme Verlag KG Stuttgart · New York ISSN 0939-978X 